\title{
Coronary artery plaques and myocardial ischaemia
}

\section{Journal Article}

Author(s):

Stolzmann, Paul; Donati, Olivio F.; Desbiolles, Lotus; Kozerke, Sebastian; Hoffmann, Udo; Alkadhi, Hatem; Scheffel, Hans

Publication date:

2011-08

Permanent link:

https://doi.org/10.3929/ethz-b-000038904

Rights / license:

In Copyright - Non-Commercial Use Permitted

Originally published in:

European Radiology 21(8), https://doi.org/10.1007/s00330-011-2097-3 


\title{
Coronary artery plaques and myocardial ischaemia
}

\author{
Paul Stolzmann • Olivio F. Donati • Lotus Desbiolles • \\ Sebastian Kozerke • Udo Hoffmann • Hatem Alkadhi • \\ Hans Scheffel
}

Received: 4 November 2010 /Revised: 11 January 2011 /Accepted: 20 January 2011 /Published online: 4 March 2011

(C) European Society of Radiology 2011

\begin{abstract}
Objective To prospectively examine coronary artery plaques as predictors of myocardial ischaemia using cardiac magnetic resonance (CMR).

Methods Fifty-two patients (46 men; age 64 \pm 10 ) with suspected coronary artery disease (CAD) referred for catheter coronary angiography (CA) underwent CMR and computed tomography coronary angiography (CTCA). All coronary segments were evaluated for morphological stenosis based on CA. Any plaque according to its composition was assessed based on CTCA.

Results Numbers of total and calcified coronary artery plaques represented the best predictors of myocardial ischae$\operatorname{mia}(\mathrm{AUC}=0.87 ;$ [95\%CI: 0.77-0.97] and $\mathrm{AUC}=0.87 ;[95 \%$ CI: $0.77-0.96$ ], respectively, $p=0.56$ ) with the total plaque number significantly higher in patients with corresponding ischaemia than those without $(p<0.01, p<0.05$ adjusted for pre-test probability and stenosis). Compared with the AUC of coronary stenosis assessment by CA (AUC $=0.90 ;[95 \%$
\end{abstract}

P. Stolzmann • O. F. Donati · H. Alkadhi · H. Scheffel Institute of Diagnostic and Interventional Radiology,

University Hospital Zurich,

Zurich, Switzerland

P. Stolzmann $(\bowtie) \cdot$ U. Hoffmann $\cdot$ H. Alkadhi $\cdot$ H. Scheffel Cardiac MR PET CT Program, Massachusetts General Hospital and Harvard Medical School,

Boston, MA, USA

e-mail: pstolzmann@partners.org

L. Desbiolles

Department of Radiology, KSSG,

St. Gallen, Switzerland

S. Kozerke

Institute for Biomedical Engineering, University and ETH Zurich,

Zurich, Switzerland
CI: $0.80-1.00]$ ), AUCs were equivalent using either the total number or the number of calcified plaques alone $(p=0.73$ and $p=0.69$ ). Multivariate logistic regression analyses demonstrated the total plaque number as an independent predictor of ischaemia (odds $+20 \%$; [95\%CI: 1.096-1.368]), improving a model including clinical probability estimates of CAD (c-statistics, 0.66 to 0.89 ).

Conclusion Coronary artery plaque number according to CTCA is a significant, independent predictor of myocardial ischaemia with similar accuracy to stenosis assessment.

Keywords Coronary artery plaque $\cdot$ Myocardial ischaemia $\cdot$ Cardiac magnetic resonance $\cdot$ Computed tomography coronary angiography $\cdot$ Coronary artery disease

\section{Introduction}

Computed tomography coronary angiography (CTCA) has become a valuable alternative to conventional coronary angiography (CA) for the reliable diagnosis or exclusion of morphological coronary artery disease (CAD) [1-4]. Nevertheless, assessment of coronary artery stenosis by both CTCA and CA insufficiently predicts myocardial ischaemia, considered to be the haemodynamic consequence of a coronary stenosis $[5,6]$.

Contrary to CA, CTCA, being a cross-sectional imaging technique, does not only provide luminographic information on the degree of coronary artery stenosis, it additionally enables the detection and characterisation of coronary plaques with good correlation with the clinical reference standard technique, intravascular ultrasound [7-9]. The association of coronary atherosclerotic plaque with myocardial ischaemia is of considerable clinical interest regarding effective risk stratification [10]. 
The purpose of this study was to prospectively examine coronary artery plaques as a predictor of myocardial ischaemia as determined by cardiac magnetic resonance (CMR).

\section{Materials and methods}

The study protocol was approved by the local institutional review board and ethics committee. All patients gave written informed consent before enrolment.

This was a single-centre, observational, cross-sectional study that was conducted prospectively, enrolling 57 patients who were referred for evaluation of suspected CAD by CA.

Exclusion criteria for CT were impaired renal function (creatinine level $>120 \mu \mathrm{mol} / \mathrm{L}, n=1$ ), known hypersensitivity to contrast medium and atrial fibrillation. Imaging with prospective electrocardiography (ECG)-gating was not performed in patients with heart rates $>70 \mathrm{bpm}(n=1)$ [11]. No medication for heart rate control was administered prior to $\mathrm{CT}$.

Exclusion criteria for CMR were contraindications to adenosine (second, $n=1$, or third degree AV block, sick sinus syndrome, symptomatic bradycardia, severe asthma or obstructive pulmonary disease, $n=1$ ) or to magnetic resonance imaging (implanted electronic devices, metallic foreign bodies in the eye, severe claustrophobia $(n=1)$, and others according to local regulations and manufacturer's recommendations). The final study population consisted of 52 patients (46 men, six women; age $64 \pm 10$, range $41-77$ ).

\section{Clinical probability assessment}

We prospectively collected data about each patient's demographics, risk factor profile (i.e. hypertension, $n=37$; hyperlipidaemia, $n=36$; diabetes, $n=10$; smoking, $n=16$, family history of CAD, $n=8$ ) and type of chest pain. Patients presented with pre-test probabilities between $3 \%$ and $94 \%$ yielding an intermediate probability of $54.7 \% \pm$ $38.4 \%$ [12].

\section{Computed tomography}

\section{Protocol}

All CT studies were performed on first-generation dualsource CT (Somatom Definition, Siemens Healthcare, Forchheim, Germany) using prospective ECG-gating. All patients received a single $2.5-\mathrm{mg}$ dose of sublingual isosorbide dinitrate (Isoket, Schwarz Pharma). Contrast agent (iopromide, Ultravist 370, Bayer AG, Zurich, Switzerland) was applied using a dual-head power injector
(Stellant, Medrad, Indianola, PA, USA) and was controlled by bolus-tracking using a region-of-interest in the ascending aorta. Contrast media injections were followed by a chaser bolus. Data were acquired in the cranio-caudal direction during mid-inspiration using the parameters: detector collimation, $2 \times 32 \times 0.6 \mathrm{~mm}$; slice acquisition, $2 \times$ $64 \times 0.6 \mathrm{~mm}$ by means of a z-flying spot; gantry rotation time, 0.33 s. Attenuation-based tube current modulation was used with a reference tube current-time product set at $190 \mathrm{mAs}$ per rotation. The data acquisition window was set at $70 \%$ of the $\mathrm{R}-\mathrm{R}$ interval; the temporal resolution was $83 \mathrm{~ms}$. CT images were reconstructed with a slice thickness of $0.6 \mathrm{~mm}$ (increment $0.4 \mathrm{~mm}$ ), using a medium smoothtissue convolution kernel (B26f). Additional reconstructions were performed using a sharp-tissue convolution kernel (B46f) to compensate for blooming artefacts. All images were transferred to an external workstation (MultiModality Workplace, Siemens Healthcare) for analysis.

\section{Image analysis}

Computed tomography data analysis was performed by two experienced radiologists who were blinded to the demographics of the patients, their clinical history and to the results from any other tests, notably $\mathrm{CMR}$ and CA. All segments with a diameter $\geq 1 \mathrm{~mm}$ at their origin were included. Vessel segments distal to occlusions were excluded from analysis. Coronary segments were defined according to a scheme proposed by the American Heart Association (AHA) [13]. The left main (segment 5) and the intermediate artery (segment 16), if present, were considered to belong to the left anterior descending coronary artery (LAD).

One reader rated the image quality of each coronary segment as being diagnostic or non-diagnostic. Degree of stenosis assessment was performed by the same reader based on comparison of the luminal diameter of the segment exhibiting stenosis with the mean luminal diameter of normal-appearing coronary sections just proximal and distal to the stenosis.

\section{Plaque number and composition}

All segments were evaluated for the presence or absence of atherosclerotic plaques by two independent readers. Coronary artery plaque was defined as any discernible structure $>1 \mathrm{~mm}^{3}$ that could be assigned to the coronary artery wall [8]. Readers ascribed the beginning and end of a plaque by visually determining discontinuity of lesions. All plaques were assessed for the presence of any plaque and non-calcified, mixed, or calcified plaque [8, 14] (window setting $500 \mathrm{HU}$ width, $200 \mathrm{HU}$ level). Noncalcified plaque was defined as any clearly discernible 
structure that could be assigned to the coronary artery wall in at least two independent image planes and had a CT density less than $130 \mathrm{HU}$ but greater than the surrounding connective tissue. Calcified atherosclerotic plaque was defined as any structure within the coronary artery wall with a density of $130 \mathrm{HU}$ or more that could be separated from the contrast-enhanced coronary lumen. When any non-calcified components were identified in the presence of calcified constituents of the same plaque, the plaque was classified as mixed. Only plaques that did not contain any calcium were classified as non-calcified. Plaque number was determined by counting the plaques on an artery basis according to plaque type. In cases of disagreement, a consensus reading between the two readers was performed.

\section{Catheter angiography}

Catheter angiography studies were evaluated by an experienced reader who was blinded to the results of CMR and CT but who was not blinded to the patients' history and symptoms. Vessels were quantitatively assessed (quantitative coronary angiography, QCA) with the use of an automated edge-detection system (Xcelera 1.2, Philips Medical Systems, Best, The Netherlands). Vessel diameter measurements were performed in at least two, preferable orthogonal image planes and included the diameter of the reference vessel (proximal and distal to the stenosis) and the minimal luminal diameter. Stenosis in each segment was classified according to the maximal luminal diameter stenosis present in each segment [15].

\section{Cardiac magnetic resonance}

\section{Protocol}

Cardiac magnetic resonance was performed on the same day and before CTCA. The patient was imaged on a 1.5-T MR whole body scanner (Intera CV; Philips Medical Systems, Best, the Netherlands) equipped with a Nova gradient system $\left(33 \mathrm{mT} / \mathrm{m}, 160\left[\mathrm{mT} \times \mathrm{m}^{-1}\right] / \mathrm{msec}\right)$ using a five-element cardiac synergy coil for signal reception. Cardiac synchronisation was performed by ECG. All data were acquired during breath-hold in end-inspiration. The true short-axis of the left ventricle was determined from long-axis scout images. Three representative short-axis sections were obtained accordingly [10]. Adenosine stress (Krenosin, sanofi-aventis, Switzerland) was applied at $140 \mu \mathrm{g} / \mathrm{min}$ per kilogram of body weight over $3.0 \mathrm{~min}$ under ECG, oxygen-saturation and blood pressure monitoring. Gadolinium-based contrast media (Gadovist 1.0; Bayer AG, Zurich, Switzerland; @ 0.1 mmol per kilogram of body weight, injection rate of $5.0 \mathrm{ml} / \mathrm{s}$, followed by a $40-$
$\mathrm{mL}$ saline flush) was administered at $2 \mathrm{~min} 30 \mathrm{~s}$ after the beginning of pharmacological stress with the acquisition of perfusion-CMR images. Ten minutes after stress perfusion imaging, a second bolus of contrast media was injected and rest perfusion images were obtained with the same orientation and position as in stress imaging. Previous knowledge driven k-t sensitivity encoding perfusion-CMR imaging was used in combination with a saturation recovery gradient-echo pulse sequence (repetition time/ echo time, 3.1/1.1 ms; flip angle, $20^{\circ}$; saturation pre-pulse delay, $110 \mathrm{~ms}$; partial Fourier sampling; acquisition window, $120 \mathrm{~ms}$; section thickness, $10 \mathrm{~mm}$; k-t factor of five with $11 \mathrm{k}-\mathrm{t}$ interleaved training profiles; effective acceleration, 3.7; three sections acquired sequentially during a single $\mathrm{R}-\mathrm{R}$ interval) [4]. Perfusion-CMR was acquired with an in-plane resolution of $2.0 \times 2.0 \mathrm{~mm}$ (reconstructed in-plane resolution of $1.25 \times 1.25 \mathrm{~mm}$ ). Ten minutes after rest perfusion, late gadolinium enhancement (LGE) images were acquired in continuous short-axis view using an inversion-recovery gradient-recalled echo MRI sequence with the following parameters: field of view, 350 $400 \mathrm{~mm}$; repetition time/echo time, 7.4/4.3 ms; inversion time, 200-350 ms; flip angle, $20^{\circ}$; matrix, $240 \times 240$; slice thickness, $10 \mathrm{~mm}$. Inversion time was chosen to null the signal from normal myocardium.

\section{Image analysis}

Perfusion stress and rest images as well as LGE images were analysed by one experienced reader, who was blinded to the patient's demographics and the results of CTCA and CA. Perfusion deficits and LGE were detected by subjective visual analysis [5]. Myocardial ischaemia was defined as (a) territories without LGE that showed a perfusion deficit during stress perfusion, but not at rest (stressinducible deficit), for three or more consecutive image frames, and (b) territories with non-transmural LGE demonstrating additional stress-inducible perfusion deficits extending larger than LGE [11]. Segments were allocated according to standard definitions [10].

\section{Statistical analysis}

Quantitative data are expressed as means $\pm \mathrm{SD}$, categorical data are given as frequencies (percentages). To prove the association of stenosis $>50 \%$ by CTCA and CA with myocardial ischaemia, two-by-two tables were fitted and Fisher's exact test was used to compare proportions.

Inter-reader reliability regarding the assessment of total plaque number and composition of plaque was assessed with $\kappa$ statistics.

To assess the association of coronary artery plaques with myocardial ischaemia, total numbers of plaques in patients 
with underlying ischaemia were compared with those in patients without ischaemia using the Mann-Whitney $U$ test and ANOVA with parameters of pre-test probability and coronary stenosis as co-variates. Univariate associations of total number of plaques, numbers of plaques according to their composition, and coronary stenosis by $\mathrm{CA}$ with myocardial ischaemia were additionally carried out through the use of receiver operating characteristic (ROC) analyses.
We performed multivariate logistic regression analyses with stepwise forward entry to determine whether the total number of coronary plaques added incremental value to the outcome variable myocardial ischaemia. The model initially included clinical probability estimates. In a second step, we added the total number of plaques as a continuous variable to the model. In a third step, we added coronary stenosis according to CA. Because co-linearity between CTCA and
Fig. 1 Conventional coronary angiography rules out significant stenosis in the right coronary artery $(R C A, \mathbf{a})$, the left anterior descending artery ( $L A D$, b), and the circumflex artery in this 71-year-old male patient. Accordingly, CT coronary angiography does not show evidence of stenosis $>50 \%$ but demonstrates numerous calcified coronary plaques in the RCA (c) and the LAD (d). Perfusion cardiac magnetic resonance indicates stress-induced ischaemia (e) in mid-ventricular septal segments (arrows) in the presence of normal rest perfusion (f)
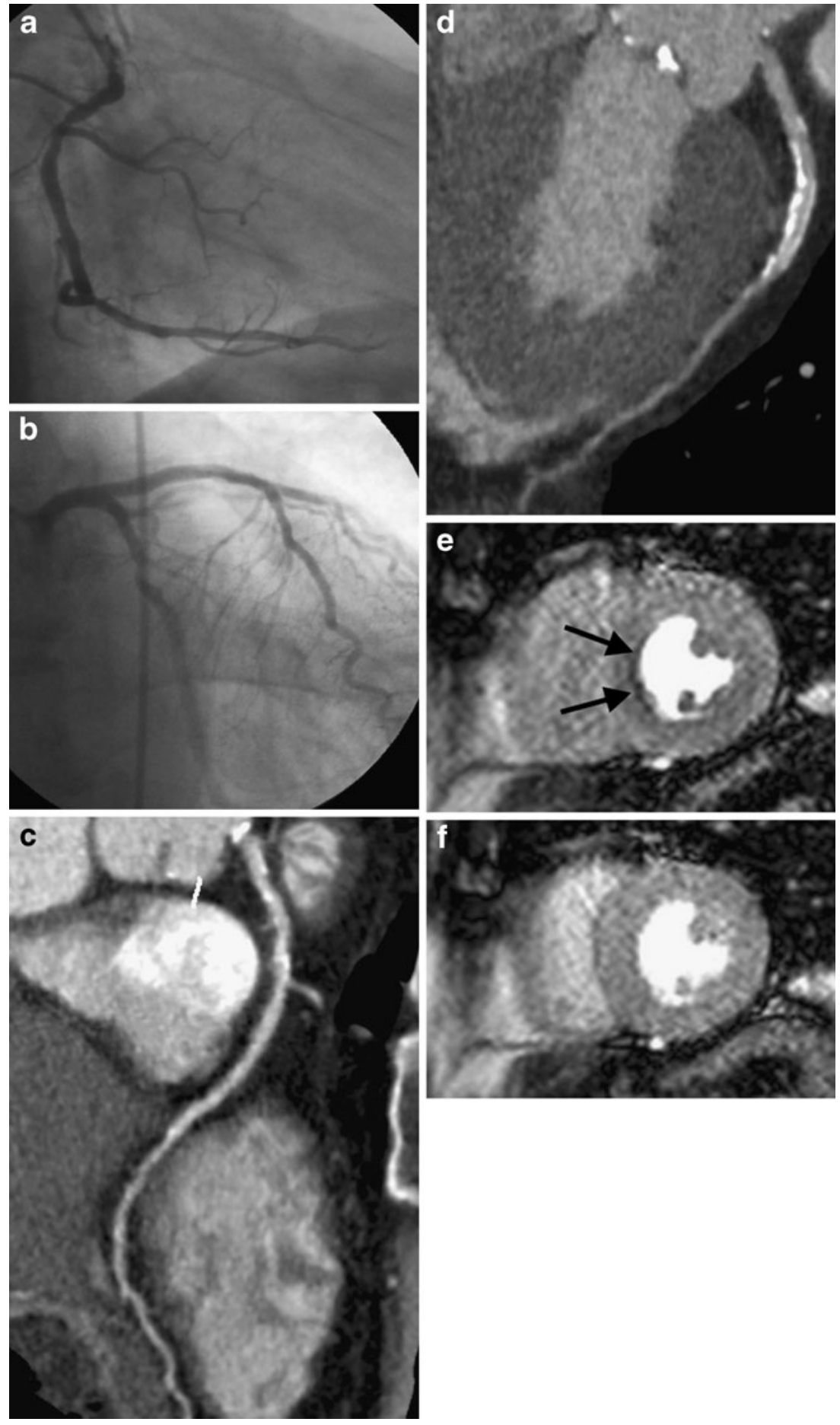


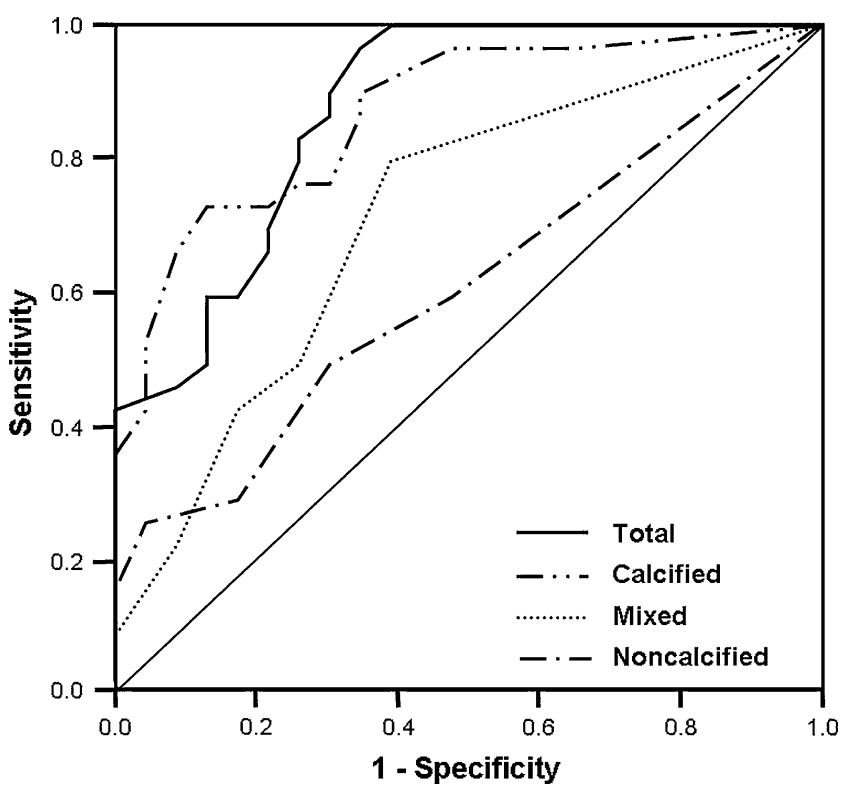

Fig. 2 Receiver operating characteristic curves for plaque numbers according to plaque composition as determined by CTCA adjusted versus myocardial ischaemia at CMR

CA regarding coronary stenosis was demonstrated by linear regression analysis $(r=0.92, p<0.001)$, only measurements derived from CA were added to the model.

The goodness-of-fit of the model was evaluated using the Hosmer-Lemeshow test and c statistics.

Statistical analysis was performed using commercially available software (SPSS, release 18.0.0; SPSS, Chicago, IL, USA). A $p$ value of $<0.05$ was considered significant.

\section{Results}

All CTCA and CMR examinations were performed within 1 day. The mean time interval between CTCA/CMR and CA was $17 \pm 8$ days (range, 1-38 days). After adenosine injection, no adverse effects leading to discontinuation of CMR were observed.

Cardiac MR image analysis revealed myocardial ischaemia in $29 / 52(56 \%)$ patients.

Inter-reader reliability for the assessment of total plaque number and composition of plaque was $\kappa=0.71$ and $\kappa=0.63$, respectively.

A total of 655 plaques (LAD, 294; RCA, 240; CX, 121) were found in $45 / 52(87 \%)$ patients. Of those, $452 / 655$ (69.0\%) were calcified (LAD, 225; RCA, 145; CX, 82), 91/ 655 (13.9\%) were non-calcified (LAD, 42; RCA, 29; CX, 20 ), and 112/655 (17.1\%) were of mixed composition (LAD, 27; RCA, 66; CX, 19). Arteries with corresponding ischemia had significantly more calcified and mixed plaques than arteries without corresponding ischemia $(p<0.0001)$.

Patients with corresponding ischaemia exhibited a significantly higher number of total plaques than those without corresponding ischaemia $(p<0.01)$ which remained significantly different $(p<0.05)$ after adjustments for pretest probability and stenosis (Fig. 1). Areas under the curves for the prediction of ischaemia were greatest and similar $(p=0.56)$ when considering the total number of plaques (AUC $=0.87$; [95\% CI: 0.77, 0.97]); and the number of calcified plaques (AUC $=0.87$; [95\%CI: 0.77, 0.96]). AUCs for the numbers of non-calcified and mixed plaques were $\mathrm{AUC}=0.60$ [95\%CI: $0.44,0.75]$ and $\mathrm{AUC}=0.71[95 \% \mathrm{CI}$ : 0.56, 0.85], respectively (Fig. 2).

Compared with the AUC regarding coronary stenosis assessment by CA (AUC $=0.90$ [95\%CI:0.80, 1.00]), AUCs were equivalent regarding either the total number or the number of calcified plaques alone $(p=0.73$ and $p=0.69$, respectively).

Table 1 summarises the association between ischaemia and the different variables in the various model steps. The Hosmer and Lemeshow test showed a good model fit (first step significance $p=0.31$, second; $p=0.57$; third, $p=0.76$ ).

Table 1 Logistic regression analyses for the prediction of ischaemia

\begin{tabular}{|c|c|c|c|c|c|c|}
\hline & Coefficient & SD & $\beta$ estimate & $95 \% \mathrm{CI}$ & $P$ & $C$ statistics \\
\hline \multicolumn{7}{|c|}{ (a) Model for the probability of ischaemia according to Diamond and Forrester } \\
\hline Risk & 0.020 & 0.008 & 1.020 & $1.005-1.036$ & $<0.05$ & 0.66 \\
\hline \multicolumn{7}{|c|}{ (b) Model for the probability of ischaemia according to Diamond and Forrester as well as total number of plaques } \\
\hline Risk & 0.022 & 0.011 & 1.023 & $1.001-1.044$ & $<0.05$ & \\
\hline Plaque $^{\mathrm{a}}$ & 0.203 & 0.057 & 1.225 & $1.096-1.368$ & $<0.001$ & 0.89 \\
\hline \multicolumn{7}{|c|}{ (c) Model for the probability of ischaemia according to Diamond and Forrester, total number of plaques, and coronary stenosis } \\
\hline Risk & 0.007 & 0.014 & 1.007 & $0.980-1.034$ & 0.63 & \\
\hline Plaque $^{\mathrm{a}}$ & 0.143 & 0.074 & 1.153 & $0.998-1.332$ & 0.05 & \\
\hline Stenosis ${ }^{\mathrm{b}}$ & 4.108 & 1.290 & 60.854 & $4.859-762.159$ & $<0.01$ & 0.93 \\
\hline
\end{tabular}

Note similarity of $C$ values regarding models (b) and (c). Plaque number ${ }^{\mathrm{a}}$ and coronary stenosis $>50 \%{ }^{\mathrm{b}}$ derived from CTCA and CA, respectively. $C A D$ coronary artery disease, $S D$ standard deviation; $C I$ confidence interval 
Multivariate logistic regression demonstrated that the total number of plaques improved the initial model including clinical probability estimates of CAD alone (c statistic, 0.66 to 0.89 ). The average increase in odds of having ischaemia by each additional plaque was $20 \%(95 \%$ CI: 1.096-1.368) when only traditional risk factor assessment is considered.

Adding the degree of stenosis improved the $c$ value of the model by 0.04 (c statistics, 0.89 to 0.93 , see Table 1), and was associated with an increased $p$ value for probability estimates and plaque numbers.

\section{Discussion}

This study analysed the relationship between coronary artery plaques and plaque composition according to CTCA with myocardial ischaemia as determined by CMR, in comparison with the prediction of ischaemia based on luminographic analysis of coronary artery stenosis. Our results indicate the total plaque number to be a significant predictor of myocardial ischaemia with an incremental value over traditional risk factor assessment which is independent of coronary stenosis assessment.

We demonstrated that the plaque numbers were higher in patients with myocardial territories showing ischaemia regardless of coronary stenosis. In our analysis, the accuracy for predicting myocardial ischaemia by the total and calcified coronary artery plaque numbers was similar to that by using coronary stenosis alone. A comparable relationship has recently been described by Schenker et al. [10], who demonstrated that the coronary artery calcium score (CAC) correlates well with myocardial ischaemia.

Although coronary artery calcium (CAC) scoring is recommended to measure the extent of calcified coronary plaques and for risk stratification [16], it does not allow for the identification of non-calcified components of atherosclerotic plaques. This is similarly reflected by the results of Schenker and colleagues [10]. Sixteen percent of their patients with a CAC of zero still showed myocardial ischaemia. In our study, we evaluated all plaques according to their different compositions by the use of data from contrast-enhanced CTCA rather than CAC.

In addition, arteries with corresponding ischemia had more plaques than arteries without ischemia. Thus, we may believe that plaque number might be helpful in ascribing functional defects to culprit arteries.

The fact that the numbers of non-calcified and/or mixed plaques were weaker univariate predictors than total and calcified plaque numbers can be attributed to the inclusion of patients [7]. It can be well speculated that non-calcified and/or mixed plaques would be better predictors in patients presenting with acute coronary syndromes.
We were able to demonstrate that coronary artery plaque burden is a significant, independent predictor of myocardial ischaemia, improving a model based on the clinical probability estimates for CAD alone [12] which holds true in patients with low to high pre-test risks. Herein, the odds of myocardial ischaemia increased by $20 \%$ for each additional plaque. Conversely, the addition of coronary stenosis by CA significantly improved neither the $\mathrm{c}$ statistics nor the goodness-of-fit of the model.

Overall, our study indicates that an elevated plaque number may be indicative of ischaemia in symptomatic patients regardless of degree of stenosis, potentially rendering subsequent stress-perfusion CMR appropriate [17].

There were a few study limitations. First, we did not determine the volume or configuration of the plaque and plaque assessment was done on a visual basis as described previously $[8,14]$. Second, statistical models only consider coronary artery stenosis $>50 \%$ as measured in one imaging plane. Third, we did not investigate patients with acute coronary syndromes which might have indicated the role of non-calcified and mixed plaques with regard to myocardial ischaemia. Fourth, our results apply to a selected patient cohort referred for CA. Furthermore, most of the patients in our cohort were male. Although we found a good accuracy for the detection of myocardial ischaemia on the basis of plaque numbers and composition in our cohort, this relationship may be different in other patient cohorts consisting, among others, of a higher percentage of women.

\section{Conclusion}

Coronary artery plaque number according to CTCA is a significant, independent predictor of myocardial ischaemia with similar accuracy to that of using coronary stenosis assessment.

\section{References}

1. Budoff MJ, Achenbach S, Blumenthal RS et al (2006) Assessment of coronary artery disease by cardiac computed tomography: a scientific statement from the American Heart Association Committee on Cardiovascular Imaging and Intervention, Council on Cardiovascular Radiology and Intervention, and Committee on Cardiac Imaging, Council on Clinical Cardiology. Circulation 114:1761-1791

2. Hendel RC, Patel MR, Kramer CM et al (2006) ACCF/ACR/ SCCT/SCMR/ASNC/NASCI/SCAI/SIR 2006 appropriateness criteria for cardiac computed tomography and cardiac magnetic resonance imaging: a report of the American College of Cardiology Foundation Quality Strategic Directions Committee Appropriateness Criteria Working Group, American College of 
Radiology, Society of Cardiovascular Computed Tomography, Society for Cardiovascular Magnetic Resonance, American Society of Nuclear Cardiology, North American Society for Cardiac Imaging, Society for Cardiovascular Angiography and Interventions, and Society of Interventional Radiology. J Am Coll Cardiol 48:1475-1497

3. Leschka S, Alkadhi H, Plass A et al (2005) Accuracy of MSCT coronary angiography with 64-slice technology: first experience. Eur Heart J 26:1482-1487

4. Weustink AC, Meijboom WB, Mollet NR et al (2007) Reliable high-speed coronary computed tomography in symptomatic patients. J Am Coll Cardiol 50:786-794

5. Hacker M, Jakobs T, Matthiesen F et al (2005) Comparison of spiral multidetector CT angiography and myocardial perfusion imaging in the noninvasive detection of functionally relevant coronary artery lesions: first clinical experiences. J Nucl Med 46:1294-1300

6. Gaemperli O, Schepis T, Valenta I et al (2008) Functionally relevant coronary artery disease: comparison of 64-section CT angiography with myocardial perfusion SPECT. Radiology 248:414-423

7. Schroeder S, Kopp AF, Baumbach A et al (2001) Noninvasive detection and evaluation of atherosclerotic coronary plaques with multislice computed tomography. J Am Coll Cardiol 37:1430-1435

8. Leber AW, Knez A, Becker A et al (2004) Accuracy of multidetector spiral computed tomography in identifying and differentiating the composition of coronary atherosclerotic plaques: a comparative study with intracoronary ultrasound. J Am Coll Cardiol 43:1241-1247

9. Achenbach S, Moselewski F, Ropers D et al (2004) Detection of calcified and noncalcified coronary atherosclerotic plaque by contrast-enhanced, submillimeter multidetector spiral computed tomography: a segment-based comparison with intravascular ultrasound. Circulation 109:14-17

10. Schenker MP, Dorbala S, Hong EC et al (2008) Interrelation of coronary calcification, myocardial ischemia, and outcomes in patients with intermediate likelihood of coronary artery disease: a combined positron emission tomography/computed tomography study. Circulation 117:1693-1700

11. Scheffel H, Alkadhi H, Leschka S et al (2008) Low-dose CT coronary angiography in the step-and-shoot mode: diagnostic performance. Heart 94:1132-1137

12. Diamond GA, Forrester JS (1979) Analysis of probability as an aid in the clinical diagnosis of coronary-artery disease. $\mathrm{N}$ Engl $\mathrm{J}$ Med 300:1350-1358

13. Austen WG, Edwards JE, Frye RL et al (1975) A reporting system on patients evaluated for coronary artery disease. Report of the Ad Hoc Committee for Grading of Coronary Artery Disease, Council on Cardiovascular Surgery, American Heart Association. Circulation 51(4 Suppl):5-40

14. Sato A, Ohigashi H, Nozato T et al (2010) Coronary artery spatial distribution, morphology, and composition of nonculprit coronary plaques by 64-slice computed tomographic angiography in patients with acute myocardial infarction. Am J Cardiol 105:930-935

15. Raff GL, Gallagher MJ, O’Neill WW, Goldstein JA (2005) Diagnostic accuracy of noninvasive coronary angiography using 64-slice spiral computed tomography. J Am Coll Cardiol 46:552557

16. Berman DS, Hachamovitch R, Shaw LJ et al (2006) Roles of nuclear cardiology, cardiac computed tomography, and cardiac magnetic resonance: noninvasive risk stratification and a conceptual framework for the selection of noninvasive imaging tests in patients with known or suspected coronary artery disease. J Nucl Med 47:1107-1118

17. Shaw LJ, Bairey Merz CN, Pepine CJ et al (2006) Insights from the NHLBI-Sponsored Women's Ischemia Syndrome Evaluation (WISE) Study: Part I: gender differences in traditional and novel risk factors, symptom evaluation, and gender-optimized diagnostic strategies. J Am Coll Cardiol 47:4-20 